\title{
SUPLEMENTACIÓN DE TERNERAS LACTANTES DOBLE PROPÓSITO EN LA ÉPOCA SECA EN EL VALLE MEDIO DEL SINÚ, COLOMBIA
}

\author{
SUPPLEMENTATION OF LACTATING CALVES DUAL \\ PURPOSE IN THE DRY SEASON IN THE MIDDLE SINU \\ VALLEY, COLOMBIA
}

\author{
Carlos Oviedo', Ángela Pastrana², Libardo Maza $^{3}$, Roger Salgado4, Oscar Vergara ${ }^{5}$
}

\begin{abstract}
${ }^{1}$ M.V.Z, Universidad de Córdoba, Facultad de Medicina Veterinaria y Zootecnia, Montería, Colombia, carlosoviedop@gmail.com ${ }^{2}$ M.V.Z, Profesional particular, Montería, Colombia, angelapastrana@hotmail.com ${ }^{3}$ M.Sc. Universidad de Córdoba, Facultad de Medicina Veterinaria y Zootecnia, Montería, Colombia, libardomaza@gmail.com ${ }^{4}$ M.Sc. Universidad de Córdoba, Facultad de Medicina Veterinaria y Zootecnia, Montería, Colombia, rodasaot@yahoo.es ${ }^{5}$ Dr.Sc, Universidad de Córdoba, Facultad de Medicina Veterinaria y Zootecnia, Montería, Colombia, overgara@sinu.unicordoba.edu.co
\end{abstract}

Rev. U.D.C.A Act. E Div. Cient. 14(1): 57 - 62, 2011

\section{RESUMEN}

El objetivo de esta investigación fue evaluar la ganancia de peso vivo y la relación beneficio - costo de terneras lactantes manejadas bajo el sistema doble propósito, suplementadas con semilla de algodón y melaza, durante la época seca en la finca Picacho, municipio de Montería, departamento de Córdoba, Colombia. Se utilizaron 26 terneras mestizas, divididas aleatoriamente en un grupo testigo (13 animales), que recibía el manejo tradicional de la finca y un grupo experimental (13 animales), que tomaban un suplemento alimenticio, a base de semilla de algodón y de melaza. Los animales, después de un periodo de adaptación de dos semanas, fueron pesados cada 14 días, durante 84 días. Para el análisis estadístico, se aplicó la prueba t de Student y análisis de varianza de medidas repetidas en el tiempo. La relación beneficio - costo, se obtuvo a través de la técnica del presupuesto parcial. El promedio de la ganancia diaria de peso difirió $(P \leq 0.05)$ entre el grupo experimental $(484 \mathrm{~g})$ y el testigo $(346 \mathrm{~g})$. Los ingresos netos generados por el tratamiento experimental fueron de 80.462 pesos y para el testigo, 58.154. El incremento en el ingreso neto fue de 22.308 pesos, con una tasa de retorno marginal de 1,73 y un beneficio económico del grupo experimental sobre el testigo de 9.346 por animal. Se concluyó que el suplemento disminuyó el efecto negativo de la época seca sobre la ganancia de peso, además, de ser una alternativa económicamente viable.
Palabras clave: Ganancia de peso, melaza, semilla de algodón, suplemento.

\section{SUMMARY}

The aim of this research was to evaluate weight gain and the relation benefit - cost of lactating calves, managed under the dual purpose system, supplemented with cottonseed and molasses during the dry season in the Picacho farm in the Municipality of Monteria, Department of Cordoba, Colombia. 26 lactating crossbred calves were used, randomly divided in a control group of 13 animals that received the traditional farm management and an experimental group of 13 animals that received also the traditional farm management and supplement food based on cottonseed and molasses. After an adaptation of two weeks the calves were weighed every 14 days during 84 days. Student t test and analysis of repeated measures was used for the statistical analysis. The relation benefit - cost was obtained through the technique of partial budget. The average daily gain differed $(P \leq 0,05)$ between the experimental group $(484 \mathrm{~g})$ and the control (346g). Net incomes generated for the experimental treatment were 80462 pesos and for the control were 58154 pesos. The increase in net income was 22308 pesos, with a marginal return rate of 1,73 and an economic benefit of the experimental group on the control group of 9346 pesos per animal. In conclusion the supplement reduced the negative 
effect of the dry season on body weight gain and represents also an economically viable alternative.

Key words: Cottonseed, molasses, supplement, weight gain.

\section{INTRODUCCIÓN}

En la Región Caribe colombiana, se presenta un déficit de alimento para el ganado durante la época seca, debido a que la oferta de pastos o de forrajes es baja, tanto en calidad como en cantidad. Esto impide a los animales cubrir sus requerimientos nutricionales de mantenimiento trayendo, como consecuencia, pérdidas de peso, bajas en la reproducción y en la producción de leche, hasta en un 50\% y la muerte en algunos casos, generando grandes pérdidas económicas a los productores (Reza et al. 2003; Tatis, 2005).

Esta situación preocupa y motiva a buscar alternativas, suministrando complementos alimenticios, como subproductos agrícolas o alimentos balanceados, para disminuir los estragos que pueda dejar en la producción.

A través del tiempo ha existido mucho interés en la búsqueda de soluciones para mejorar la alimentación en la vacada adulta, ya que, comúnmente, solo se le estima el índice productivo representado en la leche que puedan producir, dejando a un lado la cría, a la cual, se le da malos manejos alimenticios. Esto se refleja en una ganancia de peso disminuida y un bajo peso al destete, que oscila entre 90 a $130 \mathrm{~kg}$ de peso vivo, a los nueve meses de edad (Reza et al. 2003). Estos resultados, se pueden cambiar implementando alternativas de alimentación, como es la suplementación a los terneros, con la finalidad de incrementar su desarrollo en las producciones de doble propósito, predominantes en la región (Viloria, 2004).

Las investigaciones sobre suplementación han demostrado que en el período de desarrollo y de recimiento de los animales es necesario suministrar una alimentación adecuada para lograr el perfeccionamiento completo de sus sistemas orgánicos y una producción óptima cuando sean adultos.

La semilla de algodón y la melaza son subproductos agroindustriales con alta disponibilidad en la Costa Norte colombiana. La semilla de algodón representa una importante fuente de alimento para el ganado, por su alto contenido proteico (alrededor del $24 \%$ ), de fibra (cerca del $21 \%$ de fibra cruda, con un $20 \%$ de fibra detergente ácida y un $40 \%$ de fibra detergente neutra) y de concentración energética en forma de grasa (alrededor del 16\% de extracto etéreo), además de proporcionar minerales, como fósforo, magnesio y calcio (Brasi E Drouillard 2002; Corpoica, 2005). Por otra parte, la melaza es el principal subproducto de la industria de la caña de azúcar y contiene entre el 25 y el $40 \%$ de sacarosa y entre el 12 a 25\% de azúcares reductores, para un 50 a $60 \%$ de azúcares totales. Su contenido en proteína es bajo y variable, aproximadamente, del 3\% (Agudelo, 2001; Church et al. 2002).

Por lo anterior, el objetivo de este trabajo fue evaluar el comportamiento productivo y la viabilidad económica de la suplementación de terneras lactantes con una mezcla de semilla de algodón más melaza, durante la época seca.

\section{MATERIALES Y MÉTODOS}

Esta investigación, se desarrolló en la finca Picacho en la vereda Sevilla, del municipio de Montería, departamento de Córdoba, Colombia. Este municipio, se encuentra ubicado a $8^{\circ} 45^{\prime}$ de latitud Norte y $75^{\circ} 53^{\prime}$ de longitud Oeste; posee una temperatura promedio anual de $28^{\circ} \mathrm{C}$, humedad relativa del $85 \%$, precipitación promedio anual de $1600 \mathrm{~mm}$, que se presenta, principalmente, de mayo a noviembre y una altura sobre el nivel del mar de $18 \mathrm{~m}$.

En la finca, el manejo tradicional a los animales consistió en iniciar el ordeño a las 3:30 a.m. y terminarlo a las 7:00 a.m., tiempo en el que el ternero permaneció con su madre, después de ser ordeñada, para consumir la leche residual. Al finalizar el ordeño, los terneros y las vacas eran llevados a pastorear en potreros separados, exceptuando los neonatos, que acompañaban a sus madres; alrededor de las 2:00 p.m., todos los terneros eran encerrados en los corrales por el resto del día. Las pasturas de los potreros eran alrededor de un $70 \%$ de pasto Angleton (Dychanthium aristatum) y un 30\% de pasto Braquipara (Brachiaria arrecta). Al iniciar el periodo experimental, el pasto Angleton tenía un $28 \%$ de materia seca, $7 \%$ de proteína bruta, 65\% de FDN y $50 \%$ de FDA y el Braquipara, un 22\% de materia seca, 6,5\% de proteína bruta, $61 \%$ de FDN y $46 \%$ de FDA.

Para el estudio, se utilizaron 26 terneras mestizas, de diferentes proporciones de Cebú, Holstein, Pardo Suizo y Simental, en un sistema de doble propósito, con edades entre 4 a 5 meses y pesos promedios de 75,5 $\pm 2,1 \mathrm{~kg}$, a las cuales, se les daba un manejo tradicional.

Diseño experimental: Para este estudio, se utilizó un diseño completamente aleatorizado, en el que los animales se dividieron al azar en dos grupos: uno testigo (GT; 13 animales), que permanecía en pastoreo, sin recibir suplementación y otro experimental (GE; 13 animales) que, después del pastoreo tomaban $500 \mathrm{~g}$ de una mezcla de $300 \mathrm{~g}$ de semilla de algodón y $200 \mathrm{~g}$ de melaza. La semilla de algodón, se suministró entera y no fue sometida a ningún 
tratamiento adicional. Tanto la melaza como la semilla de algodón, tuvieron alta disponibilidad en los mercados locales, consiguiéndose estás en bodegas agrícolas y almacenes agropecuarios locales, a pesar de la gran demanda que tenían durante el periodo seco.

Al inicio, los animales tuvieron un período de adaptación de 14 días, a partir del cual, se realizaron los pesajes cada 14 días, utilizando una báscula ganadera mecánica de un kg de precisión, hasta finalizar el periodo experimental (84 días). El periodo experimental estuvo comprendido entre inicios de febrero e inicios de mayo.

Análisis estadístico: Para evaluar si existía diferencia estadística $(P \leq 0,05)$ para la ganancia diaria de peso entre los tratamientos durante el periodo experimental, se aplicó la prueba t de Student. Además, se efectuó un análisis de varianza de medidas repetidas en el tiempo, usando el programa estadístico SAS (2007).

Análisis económico: Para el análisis económico, se uso la técnica del presupuesto parcial propuesta por Murcia (1985).

\section{RESULTADOS Y DISCUSIÓN}

Los animales, se adaptaron fácilmente al consumo del suplemento, ya que al tercer día de iniciado el periodo de adaptación el grupo experimental ingería la totalidad de la mezcla suministrada.

La ganancia diaria de peso fue de $484 \mathrm{~g}$ para el tratamiento experimental y $346 \mathrm{~g}$ para el tratamiento testigo, logrando el primero, 138g/día de ganancia adicional de peso sobre el grupo testigo $(P \leq 0,05)$. Esta respuesta, se atribuye a que la suplementación fibroso-proteica y energética, además de contribuir a cubrir sus requerimientos, indujo un mayor desarrollo ruminal y un mejor establecimiento de su microflora, acciones que facilitaron una digestión superior de los alimentos fibrosos (Anderson et al. 1987; Garzón 2007). En igual sentido, es de esperar que haya existido una síntesis más activa de vitamina B y de proteína, a partir de compuestos nitrogenados (Canelones $\mathcal{E}$ Castejon, 2006), aspecto importante a tener en cuenta en sistemas de producción ganadera con posibilidades de utilizar los alimentos disponibles en la región.

Ganancias diarias de peso en terneros suplementados inferiores a la de este estudio han sido halladas por diferentes autores. Sandoval et al. (1993), en Venezuela, encontraron una ganancia diaria de peso de $391 \mathrm{~g}$ en terneros doble propósito en pastoreo, suplementados con $500 \mathrm{~g} /$ animal/día de alimento balanceado comercial (18\% de proteína cruda y 10\% de fibra); Alfani et al. (1996), en terneros mestizos lecheros destetados a las diez semanas en pastoreo y suplementados con alimento balanceado comercial (18\% de proteína cruda) y heno a voluntad, en Maracaibo-Venezuela (411g) y Sidibé-Anago et al. (2008), en terneros doble propósito Cebú, alimentados con heno, con torta de algodón y con melaza a voluntad, en Burkina Faso, África (149g). Igual situación fue reportada en terneros doble propósito Mpwapwa (416g), suplementados con alimento balanceado comercial a voluntad, en Tanzania (Mejía et al. 1998) y en terneros criollos Limoneros (378g) en pastoreo y suplementados con 300 g/animal/día de un alimento balanceado de $18 \%$ de proteína cruda más $2 \mathrm{~L}$ de leche, en el estado del Zulia-Venezuela (Bravo E Bracho, 2000).

Ganancias diarias de peso superiores han sido reportadas en terneros doble propósito, suplementados con 500g/ animal/día, de un alimento balanceado comercial, 559g, ó 400 g/animal/día de semilla de algodón, 583g (Martínez et al. 2001; Reza et al. 2003). Así mismo, en Sahiwal-Pakistan reportaron ganancias de $730 \mathrm{~g}$ en terneros Sahiwal x Holstein, suplementados con torta de algodón a voluntad (Jabbar et al. 2006).

Un valor similar para la ganancia de peso diaria de animales suplementados fue hallado en un estudio en el Estado de Portuguesa-Venezuela, en animales mestizos doble propósito, utilizando, como suplemento, harina de maíz a voluntad (480g) (Canelones \& Castejon, 2006) y por González-Stagnaro et al. (2006), en terneras de sistemas de producción doble propósito, suplementas con alimento balanceado comercial (462g), en el Estado de Zulia-Venezuela.

Morón-Fuenmayor et al. (1997) y Das et al. (1999) reportaron una ganancia de peso diaria similar a la de los animales sin suplementación de este estudio, en terneros mestizos Holstein no suplementados (349g), en ZuliaVenezuela y en terneros doble propósito sin suplemento, con amamantamiento restringido $(335 \mathrm{~g})$, bajo condiciones del trópico semiárido de Tanzania; sin embargo, Roldán et al. (2000) hallaron una ganancia superior (637g) en terneros Normando, sin suplementación en Tuluá, Colombia.

Los animales suplementados con alimentos balanceados comerciales, se esperaría presenten mayores ganancias de peso, porque las materias primas tienen mejores digestibilidades que los subproductos agroindustriales, pero las ganancias de peso diaria, dependen del sistema de alimentación utilizado y de la calidad de los suplementos empleados. Igualmente, la ganancia de peso en terneros va a depender del dimorfismo sexual (Cañas et al. 2008).

Al realizar el análisis de varianza de medidas repetidas en el tiempo para el peso de los animales, se registró que hubo diferencias altamente significativas entre tratamientos $(\mathrm{P} \leq 0,0001)$. En la interacción tratamiento por tiempo también se encontraron diferencias altamente significativas 


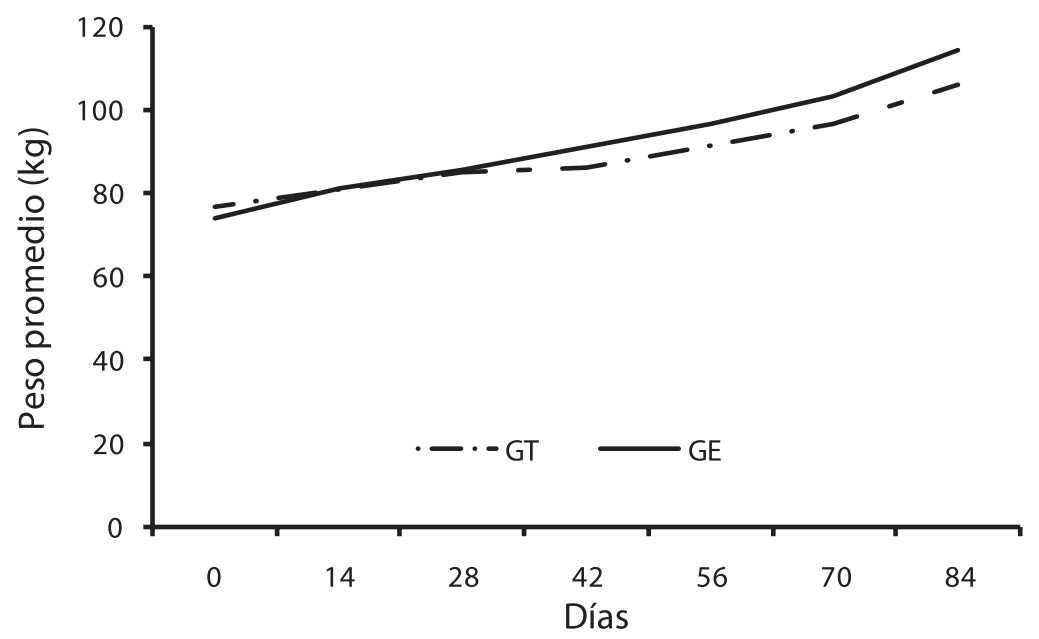

Figura 1. Promedio de los pesos de las terneras para los dos tratamientos en los diferentes pesajes del período experimental

Tabla 1. Promedios de los pesajes (kg) para el grupo testigo y experimental durante el periodo experimental.

\begin{tabular}{|l|c|c|c|c|c|c|c|}
\hline Día de pesaje & 0 & 14 & 28 & 42 & 56 & 70 & 84 \\
\hline Testigo & $76,9^{\mathrm{a}}$ & $81,5^{\mathrm{a}}$ & $85,1^{\mathrm{a}}$ & $86,5^{\mathrm{a}}$ & $91,9^{\mathrm{a}}$ & $96,5^{\mathrm{a}}$ & $106,0^{\mathrm{a}}$ \\
\hline Experimental & $74,0^{\mathrm{a}}$ & $81,2^{\mathrm{a}}$ & $85,6^{\mathrm{a}}$ & $91,1^{\mathrm{b}}$ & $97,0^{\mathrm{b}}$ & $103,2^{\mathrm{b}}$ & $114,2^{\mathrm{b}}$ \\
\hline
\end{tabular}

Medias en una misma columna con letras iguales no son estadísticamente diferentes $(P>0.05)$.

$(\mathrm{P} \leq 0,0001)$, como se puede notar en la tabla 1 ; sin embargo, el GE y el GT fueron estadísticamente iguales $(P>0,05)$, en los tres primeros pesajes, 0, 14 y 28 días y difirieron $(\mathrm{P} \leq 0.05)$, en los cuatro últimos pesajes, 42, 56, 70 y 84 días (Figura 1). Estos resultados se atribuyen, a que estos pesajes coincidieron con la época más crítica del período seco, donde existía una menor disponibilidad y calidad de las pasturas, por lo que el efecto positivo de la suplementación, en el grupo experimental, fue más evidente. Además, cabe resaltar que a partir del cuarto pesaje (42 días), el peso promedio del GT fue igual $(P>0,05)$ al peso promedio anterior (28 días) del GE, lo que confirma la superioridad de los animales suplementados respecto a los no suplementados.

En la tabla 2, se observa el resultado del análisis económico, donde se demuestra que la suplementación resulta rentable durante la época seca. La tasa de retorno marginal fue de 1,73 , es decir, que por cada peso colombiano invertido en la suplementación, se recupera en la inversión 73 centavos colombianos, con un beneficio económico de 9.346 pesos colombianos por animal; no obstante, es necesario tener en cuenta que el beneficio económico obtenido al emplear la suplementación con semilla de algodón y con melaza, se puede ver afectado por la tendencia actual que tiene la melaza a incrementar su precio, debido a su utilización para la producción de biocombustibles y a las fluctuaciones en los precios de las exportaciones de carne vacuna. Hasta el presente, lo único estable en cuanto a precios, es la semilla de algodón, por ser un subproducto de las agroindustrias de la región.

Sandoval et al. (1993) y Reza et al. (2003) hallaron una mejor tasa de retorno marginal, a la alcanzada en este estudio, en terneros suplementados con alimento balanceado comercial $(2,26)$ y con semilla de algodón $(2,35)$, en sistemas doble propósito. Por su parte, Martínez et al. (2001) encontraron una relación beneficio-costo menor 0,59 , en terneros suplementados con alimento balanceado comercial.

De estos resultados, se puede inferir que el impacto económico de la suplementación, depende del tipo de suplemento suministrado, con ventajas para los productos regionales o subproductos agroindustriales, por ser los de mayor beneficio económico para los productores, sin desconocer, que es necesario tener en cuenta el efecto de aceptabilidad que se puedan presentar por parte de los animales, donde factores, como el tipo racial utilizado y el manejo dado a los terneros, pueden también incidir (Claypool et al. 1985; Sandoval et al. 1993; Martínez et al. 2001). Existen autores que afirman que el suministro de alimento balanceado comercial en cantidad 
Tabla 2. Análisis económico mediante la técnica del presupuesto parcial por animal.

\begin{tabular}{|l|c|c|}
\hline \multicolumn{1}{|c|}{ Íltems } & Testigo & Experimental \\
\hline Costo Variable Adicionales (\$) & \multirow{3}{*}{58154} & 12962 \\
\hline Ingreso Neto (\$) & & 80462 \\
\hline Incremento en el ingreso neto (\$) & & 22308 \\
\hline Tasa de Retorno Marginal & & 1,73 \\
\hline Beneficio Económico (\$) & & 9346 \\
\hline
\end{tabular}

Se consideró que el costo de $1 \mathrm{~kg}$ de peso vivo de ternera destete era de $\$ 2.000$ y de $\$ 330$ el costo de $0,5 \mathrm{~kg}$ del suplemento.

y en calidad adecuada permite eliminar la alimentación láctea en becerros y disminuir los costos de la crianza de estos animales (Bravo \& Bracho, 2000).

Otro de los beneficios de la suplementación es que se disminuye la probabilidad de estrés al destete y permite reducir la presencia de enfermedades en el predestete (Kunkle et al. 2007).

De esta investigación, se concluye que la alimentación con suplementos proteicos y energéticos de buena calidad y en cantidades económicamente viables permite favorecer el crecimiento de las terneras y, por lo tanto, lograr una menor edad al primer parto, mejorando la eficiencia del sistema de producción ganadero.

Agradecimientos: Los autores expresan sus más sinceros agradecimientos al propietario de la Finca Picacho, en la ciudad de Montería, Colombia, por su valiosa colaboración para el desarrollo de esta investigación. Conflicto de intereses: Este manuscrito fue preparado y revisado con la participación de todos los autores, quienes declaramos que no existe ningún conflicto de intereses que pongan en riesgo la validez de los resultados presentados. Financiación: Este proyecto fue financiado por los autores y el propietario de la Finca Picacho, señor Edgardo Espitia.

\section{BIBLIOGRAFÍA}

1. AGUDELO, G. 2001. Fundamentos de Nutrición Animal Aplicada. Editorial UDEA (Medellín, Colombia). 360p.

2. ALFANI, A.; VENTURA, M.; ESPARZA, D.; DEAN, D.; VILLAR, A. 1996. Evaluación de diferentes sistemas de alimentación en becerros mestizos lecheros. Revista Facultad de Agronomía. LUZ. 13:115-134.

3. ANDERSON, K.L.; NAGARAJA, T.G.; MORILL, J.L.;
AVERY, T.B.; GALITSER, S.J.; BOYER, S.E. 1987. Ruminal microbial development in conventional or early weaned calves. Journal of Animal Science. 64:1215-1226.

4. BRASI, D.; DROUILLARD, J. 2002. Compositions and feeding value of cottonseed feed products for beef cattle. Kansas State University. 21p. Disponible desde Internet en: http://www.cottonseed.com/publications/Cottonseed\%20Feed\%20Products\%20for\%20 Beef\%20Cattle\%20-\%20KSU.pdf (con acceso el 27/09/10).

5. BRAVO, J.; BRACHO, I. 2000. Respuesta de becerros criollo limonero sometidos a diferentes niveles de suplementación. Revista Facultad de Agronomía. LUZ. 17:282-287.

6. CANELONES, C.; CASTEJON, M. 2006. Harinas de planta entera de fríjol (Vigna unguiculata) y de mazorca de maíz (Zea mays) como suplemento para becerros antes del destete. Zootecnia Tropical. 24:361-378.

7. CAÑAS, J.; RAMÍREZ, J.; ARBOLEDA, O.; OCHOA, J.; VERGARA, O.; CERÓN-MUÑOZ, M. 2008. Estimación de parámetros genéticos para peso al destete en ganado Blanco Orejinegro (BON) en el noroccidente colombiano. Revista MVZ Córdoba. 13:1138-1145.

8. CHURCH, D.G.; POND, W.G.; POND, K.R. 2002. Fundamentos de Nutrición y Alimentación de Animales. Limusa (México DF). 348p.

9. CLAYPOOL, D.W.; HOFFMAN, C.H.; OLDFIELD, J.E.; ADAMS, H.P. 1985. Canola Meal, Cottonseed, and Soybean Meals as Protein Supplements for Calves. J. Dairy Science. 68:67-70. 
10. CORPOICA. 2005. El Algodonero. Manejo integrado del cultivo en Colombia. 217p. Disponible desde Internet en: http://www.corpoica.gov.co/SitioWeb/ Archivos/Publicaciones/1GENERAL.pdf (con acceso el 27/09/10).

11. DAS, S.M.; WIKTORSSON, H.; FORSBERG, M. 1999. Effects of calf management and level of feed supplementation on milk yield and calf growth of Zebu and crossbreed cattle in the semi-arid tropics. Livestock Production Science. 59:67-75.

12. GARZÓN, B. 2007. Sustitutos lecheros en la alimentación de terneros. Revista electrónica de Veterinaria. VIII: 1695-7504. Disponible desde Internet en: http:// www.produccion-animal.com.ar/produccion_bovina de_leche/cria_artificial/84-sustitutos_leche.pdf (con acceso el 27/10/10).

13. GONZÁLEZ-STAGNARO, C.; RODRÍGUEZ-URBINA, M.; GOICOCHEA-LLAQUE, J.; MADRID-BURY, N.; GONZÁLEZ-VILLALOBOS, D. 2006. Crecimiento pre-destete en hembras bovinas doble propósito. Revista Científica. LUZ. 16:288-296.

14. JABBAR, M.A.; ANJUM, M.I.; REHMAN, S; SHAHZAD, W. 2006. Comparative efficiency of sunflower meal and cottonseed cakes in the feed of crossbred calves for meat production. Pakistan Vet. J. 26:126-128.

15. KUNKLE, B.; HOGUE, P.; JENNINGS, E.; SUMNER, S. 2007. Protein supplement may improve gains of nursing calves. SS-ANS-11. University of Florida. Disponible desde Internet en: http://edis.ifas.ufl.edu/ pdffiles/AN/AN11400.pdf (con acceso el 16/07/10).

16. MARTÍNEZ, M.; BRAVO, J.; BETANCOURT, M.; MORÁN, V. 2001. Efecto de la suplementación sobre el crecimiento de becerros mestizos en la época seca. Zootecnia Tropical. 19:31-42.

17. MEJÍA, C.; PRESTON, T.R.; FAJERSSON, P. 1998. Effects of restricted suckling versus artificial rearing on milk production, calf performance and reproductive efficiency of dual purpose Mpwapwa cattle in a semi-arid climate. Livestock Research for Rural Development 10(1). Disponible desde Internet en: http://www.Irrd. org/lrrd10/1/meji101.htm (con acceso el 29/06/10).

18. MORÓN-FUENMAYOR, O.; HUERTA-LEIDENZ, N.; ARAUJO-FEBRES, O.; MILLI, S.; ORMO, R. 1997. Efecto de la dieta sobre el desempeño biológico y económico de terneros. Revista Científica. LUZ. 7:41-46.
19. MURCIA, H. 1985. Administración de empresas asociativas de producción agropecuaria. IICA. (Costa Rica). 242p.

20. REZA, S.; CUADRADO, H.; MARTÍNEZ, J; ALVARADO, L.; ABUABARA, Y.; TORREGROSA, L. 2003. Análisis Técnico - Económico de un manejo de alimentación para bovinos lactantes en el sistema doble propósito en el Valle del Sinú. Carta Fedegan 78:82-90. Disponible desde Internet en http:// portal.fedegan.org.co/pls/portal/docs/PAGE/FNG PORTLETS/PUBLICACIONES/CARTAAFEDEGAN/ EDICIONESANTERIORES/EDION78/FONDO\%20 NACIONAL\%2078.PDF (con acceso el 12/06/10).

21. ROLDÁN, A.; PERDOMO, P.; SÁNCHEZ, H.; RAMÍREZ, M. 2000. Tecnificación del sistema de producción ganadera de doble propósito en el trópico alto andino Colombiano: amamantamiento restringido. Livestock Research for Rural Development 12(2). Disponible desde Internet en: http://www.lrrd.org/lrrd12/2/ rol122.htm (con acceso el 12/06/10)

22. SANDOVAL, E.; VALLE, A.; FLORES, R.; MEDINA, R. 1993. Crecimiento ponderal en becerros de doble propósito sometidos a un sistema integral de crianza. Zootecnia Tropical. 11:13-26.

23. SAS. 2007. SAS OnlineDoc 9.1.3. SAS Institute Inc, Cary, NC, USA.

24. SIDIBÉ-ANAGO, A.; OUEDRAOGO, G.; LEDIN, I. 2008. Effect of suckling period on calf growth and milk yield of Zebu cows. Tropical Animal Health and Production. 40:491-499.

25. TATIS, R. 2005. Amigos: Llegó la sequía y Usted qué hizo? En: Tatis, R.; Botero, L. (eds.). Génesis y consolidación del sistema vacuno en doble propósito. Produmedios (Bogotá). p.114-115.

26. VILORIA, J. 2004. La economía ganadera en el departamento de Córdoba. Documentos de trabajo sobre economía regional. Disponible desde Internet en http://www.banrep.gov.co/documentos/publicaciones/pdf/DTSER-43.pdf (con acceso el 12/06/10).

Recibido: Julio 17 de 2010

Aceptado: Abril 8 de 2011 